\title{
Spectroscopic signatures of a bandwidth-controlled Mott transition at the surface of $1 T-\mathrm{TaSe}_{2}$
}

\author{
L. Perfetti, ${ }^{1}$ A. Georges, ${ }^{2}$ S. Florens,${ }^{2}$ S. Biermann,${ }^{3}$ S. Mitrovic,${ }^{1}$ H. Berger,${ }^{1}$ Y. Tomm,,${ }^{4}$ H. Höchst,${ }^{5}$ and M. Grioni ${ }^{1}$ \\ ${ }^{1}$ Institut de Physique des Nanostructures, Ecole Polytechnique Fédérale (EPFL), CH-1015 Lausanne, Switzerland \\ ${ }^{2}$ Laboratoire de Physique Théorique de l'Ecole Normale Supérieure, \\ 24 rue Lhomond, F-75231 Paris Cedex 05, France \\ ${ }^{3}$ Laboratoire de Physique des Solides, Université Paris-Sud, Bat. 510, 91405 Orsay, France \\ ${ }^{4}$ Department of Solar Energetics, Hahn-Meitner Institut, Glienicker Str. 100, D-14109 Berlin, Germany \\ ${ }^{5}$ Synchrotron Radiation Center, University of Wisconsin, Stoughton, WI 53589-3097, USA
}

(Dated: November 17, 2018)

\begin{abstract}
High-resolution angle-resolved photoemission (ARPES) data show that a metal-insulator Mott transition occurs at the surface of the quasi-two dimensional compound $1 T-\mathrm{TaSe}_{2}$. The transition is driven by the narrowing of the Ta $5 d$ band induced by a temperature-dependent modulation of the atomic positions. A dynamical mean-field theory calculation of the spectral function of the halffilled Hubbard model captures the main qualitative feature of the data, namely the rapid transfer of spectral weight from the observed quasiparticle peak at the Fermi surface to the Hubbard bands, as the correlation gap opens up.
\end{abstract}

PACS numbers: 71.30.+h,79.60.Bm,71.45.Lr,71.10.Fd

Electronic correlations can modify the electronic structure of solids not only quantitatively, but also qualitatively, inducing new broken-symmetry phases which exhibit charge, spin or orbital-order, and more exotic states in low dimensions. One of the most notable consequences of electronic correlations is the much studied metal-insulator (M-I) Mott transition [1, 2]. Recently, new theoretical approaches have considerably extended our understanding of this fundamental problem [2, 3].

Many physical properties indirectly reflect the dramatic rearrangement of the electronic structure at the transition. Photoelectron spectroscopy, which probes the single-particle spectral function, can provide a direct view of such changes [4, 5, 6]. However, comparing samples with different compositions faces materials problems like stoichiometry, defects, and disorder. A quantitative analysis is further complicated by the known surface sensitivity of the technique [7] 8]. An ideal experiment would record the energy and momentum-dependent spectrum, while tuning the crucial $(W / U)$ parameter ( $U$ is the onsite Coulomb correlation energy; $W$ is the bandwidth) in the same single crystal sample. Remarkably, it is possible to approach this ideal situation exploiting the occurrence of modulated structures (charge-density-waves; CDWs) in appropriate low-dimensional systems. There, the lattice distortion modulates the transfer integrals and therefore modifies the bandwidth. In materials that are close enough to a Mott transition, the reduced bandwidth may lead to an instability. There are strong indications for this scenario in the layered chalcogenide $1 T-\mathrm{TaS}_{2}$, which presents a sharp order-of-magnitude increase of the resistivity at $\mathrm{T}=180 \mathrm{~K}[\underline{9}, 10]$, with a strong rearrangement of the electronic states 11, 12, 13, 14, 15]. However, the complex phase diagram of the $\mathrm{CDW}$ in $1 T-\mathrm{TaS}_{2}$ affects the electronic transition, which cannot be considered as a typical Mott transition.

Isostructural and isoelectronic $1 T-\mathrm{TaSe}_{2}$ exhibits a similar CDW, but only one phase below $\mathrm{T}_{C}=475 \mathrm{~K}$. Its electrical resistivity remains metallic - albeit rather large - to very low temperatures [9], suggesting that the Se compound lies further from the instability than the $\mathrm{S}$ analog. Nevertheless, a transition could still occur at the crystal surface, where the $U / W$ ratio is expected to be larger as a result of smaller screening and coordination. The surface sensitivity of ARPES is ideal to investigate such an inhomogeneous state. In this Letter we present evidence for a Mott transition at the surface of $1 T$ $\mathrm{TaSe}_{2}$. High-resolution temperature-dependent ARPES shows for the first time the disappearance of the coherent quasiparticle signatures at the Fermi surface and the opening of a correlation gap. These results are qualitatively well described by a dynamical mean-field (DMFT) calculation, and provide new insight into the spectral properties of the MI transition.

$1 T-\mathrm{TaSe}_{2}$ has a layered structure, with the $d^{1} \mathrm{Ta}$ atoms in a distorted octahedral environment. Adjacent layers interact weakly through van der Waals gaps, and all physical properties exhibit a strong anisotropy. A threefold CDW develops below $T_{C}=475 K$, with a commensurate $\sqrt{13} \times \sqrt{1} 3$ superstructure, analogous to the much studied low-temperature CDW phase of $1 T$ $\mathrm{TaS}_{2}[9]$. In real space the CDW corresponds to a modulation of the atomic positions, within a $13 \mathrm{Ta}$ atoms unit, in the so-called 'star-of-David' configuration. Extended Huckel calculations [16] suggest that the CDW splits the Ta $d$ conduction band into subbands which contain a total of 13 electrons per unit cell. Two subbands, carrying 6 electrons each, are filled and lie below the Fermi level. 
The Fermi surface is formed by a half-filled subband carrying the 13th electron. The opening of a correlation gap in this subband is responsible for the resistivity jump in (bulk) $1 T-\mathrm{TaS}_{2}[10$.

We performed high-resolution ARPES measurements in Lausanne and at the PGM beamline of the SRC, University of Wisconsin. The energy and momentum resolution were $\Delta \mathrm{E}=10 \mathrm{meV}$ and $\Delta k= \pm 0.02 \AA^{-1}$, and the Fermi level location was determined with an accuracy of $\pm 1 \mathrm{meV}$ by measuring the metallic edge of a polycrystalline gold reference. Single crystal samples grown by the usual iodine transport technique were characterized by Laue diffraction and resistivity measurements, which confirmed the assignment to the $1 T$ polytype. They were mounted on the tip of a closed-cycle refrigerator and cleaved at a base pressure of $1 \times 10^{-10}$ torr. We did not observe any sign of surface degradation or contamination over a typical 8 hours run.

Figure 1 shows ARPES intensity maps $(\mathrm{h} \nu=21 \mathrm{eV})$ measured at $300 \mathrm{~K}$ and $70 \mathrm{~K}$ along the high-symmetry $\Gamma \mathrm{M}$ direction of the hexagonal Brillouin zone (BZ). A higher photon energy $(\mathrm{h} \nu=50 \mathrm{eV})$ yields similar results. The maps correspond to the same CDW phase, as confirmed by low-energy electron diffraction (LEED), but exhibit remarkable differences. At $300 \mathrm{~K}$ the narrow topmost Ta $d$ subband crosses the Fermi level at $k_{F_{1,2}}$ $\sim \pm 1 / 4 \Gamma M$, in good agreement with band structure calculations [17]. The filled CDW subbands are visible at $\sim 0.3 \mathrm{eV}$ and, with lower intensity at larger binding energies $(\sim 0.8 \mathrm{eV})$ and momenta. The overall dispersion of the Ta $d$ band is influenced by the CDW superlattice, as will be discussed elsewhere. The parabolic band with a maximum at $\Gamma$ and $\sim 0.5 \mathrm{eV}$ is a Se $p$ band. At $70 \mathrm{~K}$ the Ta $d$ spectral weight is narrower and clearly removed from $E_{F}$, and a gap has appeared.

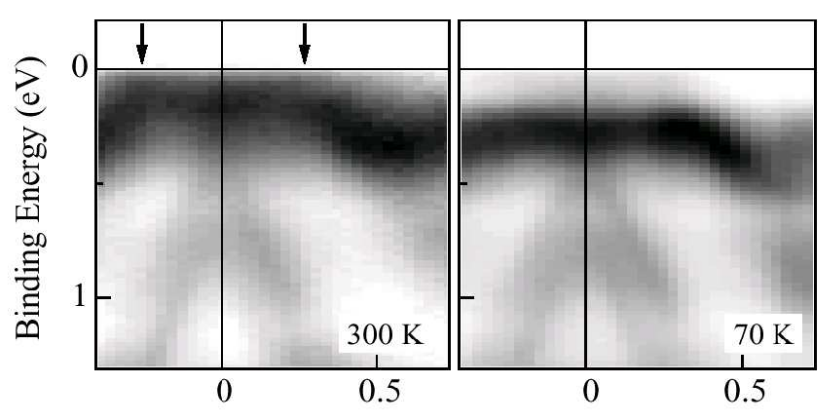

Wave vector (fraction of $\Gamma \mathrm{M})$

FIG. 1: ARPES intensity maps of $1 T-\mathrm{TaSe}_{2}$ at $\mathrm{T}=300$ $\mathrm{K}$ (left) and $\mathrm{T}=70 \mathrm{~K}$ (right) measured along the $\Gamma \mathrm{M}$ highsymmetry direction $(\mathrm{h} \nu=21 \mathrm{eV})$. The arrows mark Fermi level crossings by the Ta 'd' band at $300 \mathrm{~K}$.

Bulk sensitive properties and surface sensitive LEED data rule out a structural phase transition between 300 $\mathrm{K}$ and $70 \mathrm{~K}$. The large spectral changes are therefore the consequence of an electronic surface transition. Signa- tures of a surface gap in $1 T-\mathrm{TaSe}_{2}$ were previously observed at $70 \mathrm{~K}$ by scanning tunneling spectroscopy (STS) [18. We characterized this transition by temperaturedependent measurements of the shallow Ta $4 f$ core levels, which exhibit a CDW-induced fine structure [11, 19]. We find (not shown) that between $300 \mathrm{~K}$ and $70 \mathrm{~K}$ the CDW-split components sharpen, and their energy separation increases by $40 \mathrm{meV}$, in agreement with lowerresolution data 20]. We conclude that the CDW amplitude and the corresponding lattice distortion are larger at the lower temperature.

The increased distortion reduces the overlap between the 'cluster orbitals' which form the basis of the band structure in the CDW phase. Calculations which explicitely account for this effect are not available, but the $(W / U)$ ratio is certainly reduced, possibly below the critical value for the M-I transition. This is confirmed by an inspection of the ARPES signal at $k=k_{F_{1}}$ (Fig. 2a), which reveals a sudden loss of intensity near $E_{F}$ below $\sim 260 \mathrm{~K}$, and the appearence of a strong signal centered at $0.26 \mathrm{eV}$. All spectra were normalized to the same integrated area. The intensity at $E_{F}$ (Fig. 2b) exhibits a sharp step around $260 \mathrm{~K}$, and a further linear decrease at lower temperature. The intensity and the spectral lineshape (not shown) are recovered upon heating, but only at higher temperatures. The large hysteresis $(\sim 80 K)$ suggests a first-order transition [21].

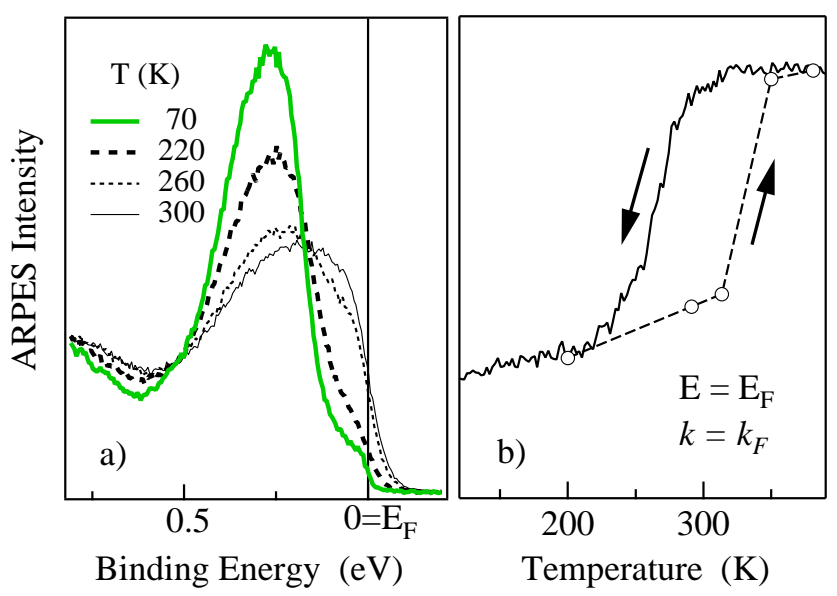

FIG. 2: a) ARPES spectra measured at $k=k_{F_{1}}$ between $300 \mathrm{~K}$ and $70 \mathrm{~K}$; b) Temperature dependence of the ARPES signal at the Fermi surface, showing a sharp break, and a large hysteresis.

In Fig. 3, following common practice in ARPES work on the cuprates, we symmetrized the spectra of Fig. 2a around $E_{F}$. This procedure removes the perturbing effect of the Fermi distribution on the intrinsic temperature dependence of the spectral function $A\left(k_{F}, \omega, T\right)$. The $300 \mathrm{~K}$ spectrum exhibits a broad $(\sim 1 \mathrm{eV})$ incoherent background and a weak quasiparticle (QP) feature at $E_{F}$. The QP signal disappears at lower tempera- 
ture, and spectral weight is transferred to the lower and - as inferred by symmetry - upper sidebands, representing the lower (LHB) and upper (UHB) Hubbard subbands. The integrated intensity is conserved. We emphasize that the width $(\sim 100 \mathrm{meV})$ of the central peak is much larger than expected for a coherent quasi-particle at the Fermi surface of a 'good' metal. Clearly, in the 220-260 K temperature range, the corresponding excitations are heavily scattered, and their lifetime is short. This 'bad metal' character is consistent with the broad maximum and large value of the electrical resistivity of bulk $1 T-\mathrm{TaSe}_{2}$ at $250-300 \mathrm{~K}[\underline{9}]$.

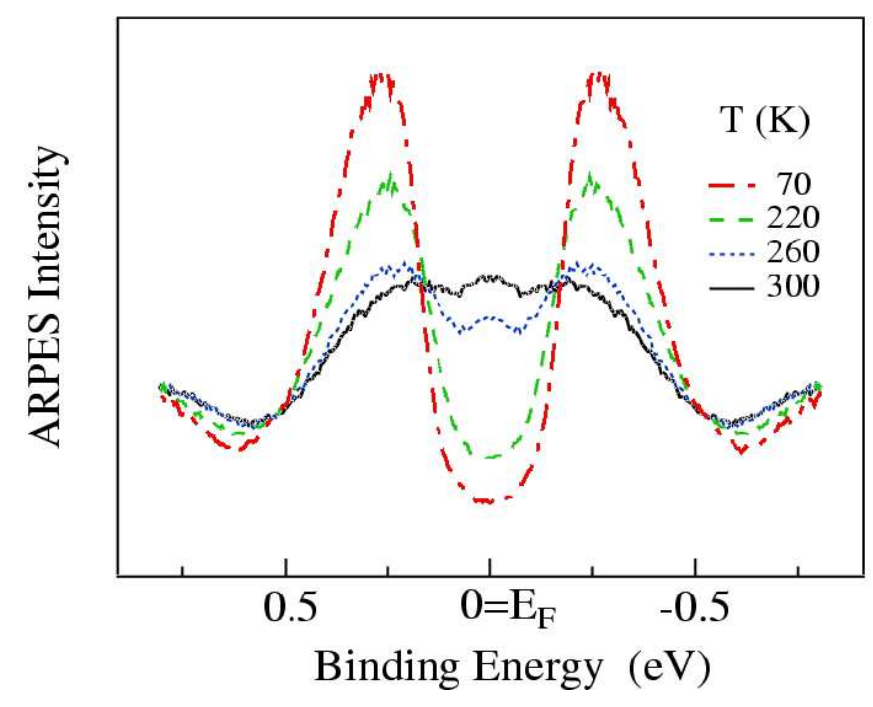

FIG. 3: Temperature-dependent ARPES spectral function at $k=k_{F_{1}}(\mathrm{~h} \nu=21 \mathrm{eV})$. The spectra have been obtained from the raw spectra $\mathrm{I}(\mathrm{E})$ by symmetrization around $E_{F}$ : $\mathrm{I}^{*}(\mathrm{E})=\mathrm{I}(\mathrm{E})+\mathrm{I}(-\mathrm{E})$.

The spectra of Fig. 3 are qualitatively consistent with the changes expected at the Mott transition. In order to substantiate this, we have calculated $A\left(k_{F}, \omega ; T\right)$ for a one-band Hubbard model at half-filling, within the dynamical mean-field theory (DMFT) framework 3|. The "iterated perturbation theory" approximation 22] was used, and checks were made using Quantum Monte Carlo and the maximum entropy method. The Coulomb term $U$ was set at $0.52 \mathrm{eV}$, equal to the energy separation between the LHB and UHB features in Fig. 3. A semicircular density of states was used, with a bandwith $W$ assumed to depend linearly on temperature between 300 $\mathrm{K}(W=0.50 \mathrm{eV})$ and $70 \mathrm{~K}(W=0.36 \mathrm{eV})$. These values are only indicative, and are not the result of a specific attempt to find an optimum fit to the data, but we note that the overall magnitude of $W$ is consistent with the dispersions observed in Fig.1.

The high-temperature $(300 \mathrm{~K}, 260 \mathrm{~K})$ calculated spectra (Fig. 4) correspond to a correlated metal in the incoherent regime, with a broad low-energy peak and two intense Hubbard sidebands. The central peak is strongly reduced at $220 \mathrm{~K}$, and at $70 \mathrm{~K}$ it has disappeared completely, leaving two sharp features centered at $\pm U / 2$ and separated by a real gap. The overall shape of the spectra is in good qualitative agreement with the data, as well as the dramatic transfer of spectral weight that takes place between the central peak and the Hubbard bands as the temperature is lowered. We observe that in both theory and experiment the spectral weight accumulated in the insulator near the maximum of the HB sidebands comes mainly from the QP central peak, but that a small fraction also comes from energies above $0.5 \mathrm{eV}$. This results in two energies at which all spectra approximately cross. The data provide the first direct momentum-resolved observation of two of the key predictions of DMFT regarding the one-particle spectrum through the Mott transition, namely the three-peak structure in the metallic state 22 and the large transfers of spectral weight from the metal to the insulator [23, 24, 25]

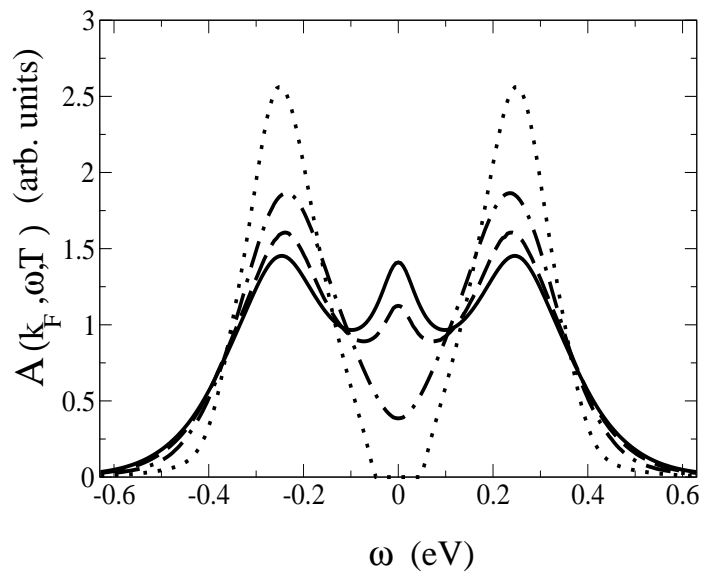

FIG. 4: Temperature-dependent spectral function $A\left(k_{F}, \omega\right)$ of the half-filled Hubbard model calculated within DMFT for the temperatures of Fig. 4. $\mathrm{U}$ was fixed at $\mathrm{U}=0.52 \mathrm{eV}$, and the bandwidth is chosen as: $\mathrm{W}=0.50 \mathrm{eV}$ at $300 \mathrm{~K}$ (solid); 0.48 $\mathrm{eV}$ at $260 \mathrm{~K}$ (dash); $0.44 \mathrm{eV}$ at $220 \mathrm{~K}$ (dash-dot); and $0.36 \mathrm{eV}$ at $70 \mathrm{~K}$ (dot).

The comparison of theory and experiment also reveals some differences. Unlike the calculated spectra, the intensity at $E_{F}$ is never completely suppressed in the experimental spectra. This signal could originate from the underlying metallic bulk, or from surface inhomogeneities (metallic 'patches') observed by STS [18]. It could also indicate that the tails of the LHB and UHB overlap slightly, a passibility which was considered in a different context 26]. Another quantitative difference is the sharper separation between the QP peak and Hubbard bands in the calculation. This is a known feature of DMFT which is likely to be weakened as dimensionality is lowered. Recent work in particular [27] suggests that long-wavelength charge modes partially fill the preformed gap.

From a theoretical standpoint, the main discrepancy concerns the value of the critical temperature at which 
the first-order metal insulator transition is observed. Indeed, with the observed value of $U$, the simple one-band Hubbard model treated within DMFT would have a firstorder transition at $\mathrm{T} \simeq U / 80 \simeq 90 \mathrm{~K}$, a factor of three smaller than observed experimentally. The trajectory in the $(\mathrm{T},(W / U))$ space used in the theoretical calculation does not intersect the first-order transition line, so that theory would interpret the spectral changes as due to a rapid crossover between a bad metal and a Mott insulator. On the other hand the experimental observation of a hysteresis does suggest a real transition. In a purely electronic model, it is known that orbital degeneracy does lead to increased $T_{c}$ values [28]. However, DMFT model calculations including the whole $d$ manifold, show a significant increase in $T_{c}$ only when the first subband is much closer to the Fermi level than in the experiment. Thus, it is unlikely that orbital degeneracy could explain the increased $T_{c}$. The inadequacy of a purely electronic model is also suggested by the large width of the low-energy QP. In a purely electronic model, this can only be accounted for if the temperature is significantly larger than the critical temperature for the metalinsulator transition.

The observation of a broad peak and a true transition strongly suggests that the coupling to lattice degrees of freedom plays an important role, as in fact expected in a CDW compound. Indeed, it has been shown in a simple model that the coupling to the lattice can lead to an increase in $T_{C}$ 29]. Also, it is possible that the ob- served hysteresis results from differences in the pinning of the CDW upon heating and cooling. Obviously, further investigation - both theoretical and experimental is required to clarify these issues.

In summary, we presented momentum-resolved highresolution ARPES data which illustrate the spectral consequences of a bandwidth-controlled surface Mott transition in $1 T-\mathrm{TaSe}_{2}$. The transition from a bad-metal, characterized by a largely incoherent spectrum, to a correlated insulator is qualitatively captured by a DMFT calculation for the half-filled Hubbard model. Quantitative differences between theory and experiment suggest that the model should be extended to include the coupling to lattice degrees of freedom, in order to provide a more accurate description of electronic transitions in such CDW materials.

We acknowledge R. Gaal for the resistivity measurements, correspondence with P. Aebi, E. Canadell, F. del Dongo, and the support of G. Margaritondo. This work has been supported by the Swiss NSF through the MaNEP NCCR. A.G is grateful to F.Mila for discussions and hospitality at UNIL-Lausanne. A.G, S.F and S.B are most grateful to KITP-UCSB for the warm hospitality during the final stage of this work, where it was supported in part by NSF under Grant PHY99-07949, and also acknowledge computing time at IDRIS-CNRS under project 011393. The Synchrotron Radiation Center, University of Wisconsin-Madison, is supported by NSF under Award No. DMR-0084402.
[1] N.F. Mott, Metal-Insulator Transitions (Taylor and Francis, London 1990).

[2] M. Imada, A. Fujimori and Y. Tokura Rev. Mod. Phys. 701039 (1998).

[3] For a review, see: A. Georges, G. Kotliar, W. Krauth and M. Rozenberg, Rev. Mod. Phys. 6813 (1996).

[4] A. Fujimori et al. Phys. Rev. Lett. 69, 1796 (1992).

[5] H.D. Kim et al. cond-mat-0108044 (unpublished); J. W. Allen private communication.

[6] S. Shin et al., J. Phys. Soc. Jpn. 64, 1230 (1995).

[7] K. Maiti et al. Europhys. Lett. 55 ,246 (2001); K. Maiti, $\mathrm{PhD}$ thesis (unpublished)

[8] S. Suga and A. Sekiyama J. El. Spectr. Rel. Phenom 124 $81(2002)$.

[9] J.A. Wilson, F.J. DiSalvo, and S. Mahajan, Adv. Phys. 24, 117 (1975).

[10] P. Fazekas and E. Tosatti, Philos. Mag. B 39, 229 (1979).

[11] R.A. Pollak et al., Phys. Rev. B 24, 7435 (1981).

[12] N.V. Smith, S.D. Kevan, and F.J. DiSalvo, J. Phys. C: Solid State Phys. 18, 3175 (1985).

[13] R, Manzke et al., Europhys. Lett. 8, 195 (1989).

[14] R. Claessen et al., Phys. Rev. B. 41, 8270 (1990).

[15] F. Zwick et al., Phys. Rev. Lett. 81, 1058 (1998).

[16] M.-W. Whangbo and E. Canadell, J. Am. Chem. Soc. 114, 9587 (1992).

[17] P. Aebi et al., J. El. Spectr. Rel. Phenom 117-118 433 (2001).
[18] O. Shiino et al., Appl. Phys. A 66, S175 (1998).

[19] H.P. Hughes and J.A. Scarfe, Phys. Rev. Lett. 74, 3069 (1995).

[20] H.P. Hughes, and R.A. Pollak, Philos. Mag. 34, 1025 (1976).

[21] A recent ARPES work on $1 T-\mathrm{TaSe}_{2}$ (K. Horiba et al, Phys. Rev. B 66073106 (2002)) reported metallic behavior at $\mathrm{T}=20 \mathrm{~K}$. It is our experience that defects/disorder rapidly distroy CDW transitions in layered materials. We speculate that subtle sample-related issues may have prevented the observation of the transition.

[22] A. Georges and G. Kotliar, Phys. Rev. B 456479 (1992).

[23] A. Georges and W. Krauth, Phys. Rev. B 487167 (1993).

[24] M.J. Rozenberg, G. Kotliar and X. Y. Zhang, Phys. Rev. B 4910181 (1994).

[25] For an early discussion of spectral weight transfers in the cerium $\alpha-\gamma$ transition, see: L. Z. Liu et al., Phys. Rev. B 458934 (1992).

[26] D.J. Thouless, J. Phys. (Paris) Colloq. 37, C4-349 (1976).

[27] S. Florens and A. Georges unpublished.

[28] S. Florens, A. Georges, G. Kotliar and O. Parcollet cond-mat 0205263 to appear in Phys. Rev. B (2002).

[29] P. Majumdar and H. R. Krishnamurthy Phys. Rev. Lett. 731525 (1994). 